\title{
ABUNDANCE EFFECTS FOR THE AO-G2 STARS \\ IN THE GENEVA SYSTEM
}

\author{
B. HAUCK \\ Institut d'Astronomie de l'Université de Lausanne et \\ Observatoire de Genève, Switzerland
}

\begin{abstract}
The abundance effects on the tri-dimensional representation for the A0-G2 stars in the Geneva system are reviewed. B2-V1 and $d$ are not affected by the Am characteristic, but B2-V1 is too blue (blocking effect on V1) and $d$ smaller for the Ap stars. For B2-V1 $\geqslant 0.230$, we have a residual effect $\Delta(\mathrm{B} 2-\mathrm{V} 1)=1.20\left(\Delta m_{2}^{*}+0.060\right)$. $\mathrm{d}$ is also affected and the residual effect is $\Delta d=-0.4$ $\Delta m_{2}$ for $\Delta m_{2} \geqslant-0.060$ and $\Delta d=-1.1 \Delta(\mathrm{B} 2-\mathrm{V} 1)-0.024$ for $\Delta m_{2}<-0.060$.

The abundance effects on the relations between B2-V1 and the parameters of temperature in other systems are studied.
\end{abstract}

\section{Introduction}

A tri-dimensional representation for the A0-G2 stars of luminosity classes V to III was presented some years ago (Hauck 1968, 1973a). The following parameters are used:

$$
\begin{aligned}
& \mathrm{B} 2-\mathrm{V} 1 \text { as temperature parameter } \\
& \begin{array}{l}
d=(\mathrm{U}-\mathrm{B} 1)-1.430(\mathrm{~B} 1-\mathrm{B} 2) \text { as luminosity parameter } \\
m_{2}=(\mathrm{B} 1-\mathrm{B} 2)-0.457(\mathrm{~B} 2-\mathrm{V} 1) \text { as blanketing parameter }
\end{array}
\end{aligned}
$$

and $\quad[\mathrm{Fe} / \mathrm{H}]=6.830 \Delta m_{2}+0.203 \quad$ for $[\mathrm{Fe} / \mathrm{H}]>-1.0$

$$
\pm 0.16 \pm 0.961 \quad \pm 0.097
$$

with $\quad \Delta m_{2}=m_{2}($ star $)-m_{2}$ (Hyades)

A review of the properties of the diagram $d$ vs B2-V1 was given by Hauck (1975a). On this occasion an extension to the supergiants was proposed. Using such a diagram it is possible to distinguish the various classes of luminosity.

The colour index B2-V1 is well correlated with $\Theta_{\text {eff }}$ or with the spectral type. A recent calibration of $\mathrm{B} 2-\mathrm{V} 1$ for the main-sequence stars is given in Table I of Hauck and Magnenat (1975). The values of $\Theta_{\text {eff }}$ adopted for this calibration are taken respectively in the papers of Schild et al. (1971) and Oke and Conti (1966) for the first calibration and in the paper of Morton and Adams (1968) for the second. Calibrations for parameters of temperature in other photometric systems are also given in this table.

In the present paper we have examined abundance effects firstly on B2-V1 and $d$ and secondly on the relations between $\mathrm{B} 2-\mathrm{V} 1$ and the parameters of temperature in other systems. 


\section{Abundance Effects on B2-V1}

\subsection{StaRS WITH B2-V1 $<0.230$ (A0-F5)}

In this range of spectral types, we have the Ap stars and the Am stars. For the latter Hauck and van 't Veer (1970) have shown that no effect was present on B2-V1. On the other hand, for the Ap stars, Gerbaldi et al. (1974) have shown that B2-V1 is too blue and that this effect is due to a blocking effect on V1. B2-G is practically not affected by this effect and seems for Ap stars a good parameter of temperature. The following relation for normal stars was derived between B2-V1 and B2-G:

$$
\mathrm{B} 2-\mathrm{G}=1.386(\mathrm{~B} 2-\mathrm{V} 1)-0.437
$$

\subsection{StaRs With B2-V1 $>0.230$ (F5-G2)}

In this range of spectral types, we have a residual effect of blanketing on B2-V1, if $\Delta m_{2}$ $\leqslant-0.060$. This effect is given by

$$
\Delta(\mathrm{B} 2-\mathrm{V} 1)=1.20\left(\delta m_{2}+0.060\right)
$$

$\delta m_{2}$ is the value determined before the correction on B2-V1, $\Delta m_{2}$ is the value obtained after the blanketing correction on B2-V1 and it is this value which is correlated with $[\mathrm{Fe} / \mathrm{H}]$.

\section{Abundance Effects on $d$}

For the Am stars no abundance effects were detected on $d$ (Hauck, 1967), while for the Ap stars, Gerbaldi et al. (1974) have shown that $d$ is affected by the Ap characteristic, in the sense that many Ap stars have a smaller Balmer discontinuity (or a $d$ value) than normal stars. This effect is certainly correlated with the blocking effect on V1 (Hauck $1975 b$ ). For stars with B2-V1 $>0.230$, we have found (Hauck 1973a) that the residual blanketing effect on $d$ is

$$
\begin{aligned}
& \Delta d=-0.4 \Delta m_{2} \text { for } \Delta m_{2} \geqslant-0.060 \\
& \Delta d=-1.1 \Delta(\mathrm{B} 2-\mathrm{V} 1)-0.24 \text { for } \Delta m_{2}<-0.060
\end{aligned}
$$

\section{Abundance Effects on the Relations between B2-V1 and Temperature Parameters in Other Photometric Systems}

Hauck and Magnenat (1975) have given for the main-sequence stars the relations between $\mathrm{B} 2-\mathrm{V} 1$ and $\mathrm{B}-\mathrm{V}, \mathrm{R}-\mathrm{I}, \boldsymbol{b}-y, \mathrm{H} \beta,(\mathrm{G}-\mathrm{I})_{6},(\mathrm{~B}-\mathrm{I})_{6}, \mathrm{Y}-\mathrm{V}$ and $\mathrm{Y}-\mathrm{S}$ parameters belonging respectively to $U B V R I, u v b y \beta, 6$-colour and Vilnius systems.

In the present study, we have examined whether these relations are also valid for Am, Ap and subdwarf stars. For Am stars we have chosen only those with known spectral 
types (Hauck, 1973b) and for Ap stars those belonging to the Osawa catalogue (1965). The subdwarfs are principally stars belonging to the lists of Cayrel (1968) and Sandage and Eggen (1959).

The result of our examination is given in Table I. It is important to note that it is only an indication concerning the validity (or the non-validity) of a relation between two colour indices and not an indication whether or not a blanketing effect is present on these indices.

\section{TABLE I}

\begin{tabular}{llllllll}
\hline $\begin{array}{l}B 2 \ldots 1 \text { vs: } \\
\text { for }\end{array}$ & $B-V$ & $R-I$ & $b-y$ & $B-I$ & $G-I$ & $Y-V$ & $Y-S$ \\
\hline $\mathrm{Am}$ & $\bullet$ & $\bullet$ & 0 & 0 & $\bullet$ & $\bullet$ & $\bullet$ \\
$\mathrm{Ap}$ & $\bullet$ & 0 & 0 & 0 & 0 & $\bullet$ & $\bullet$ \\
Subwarfs & $\bullet$ & $\bullet$ & 0 & $\bullet$ & $\bullet$ & 0 & $\bullet$ \\
\hline \\
$\begin{array}{l}\text { the relation is not valid } \\
\text { the relation is valid }\end{array}$
\end{tabular}

\section{References}

Cayrel, R.: 1968, Astrophys. J. 151, 1005.

Gerbaldi, M., Hauck, B., and Morguleff, N.: 1974, Astron. Astrophys. 30, 105.

Hauck, B.: 1967, Publ. Obs. Genève, No. 74.

Hauck, B.: 1968, Publ. Obs. Genève, No. 78.

Hauck, B.: 1973a, in B. Hauck and B. E. Westerlund (eds.) Problems of Calibration of Absolute Magnitudes and Temperature Scales, IAU Sy $m p$. 54, 117.

Hauck, B.: 1973b, Astron. Astrophys. Suppl. 10, 385.

Hauck, B.: 1975a, in A. G. D. Philip (ed.), Multicolor Photometry and the Theoretical HR Diagram (in press).

Hauck, B.: 1975b in C. Jaschek (ed.), Physics of Ap Stars, IAU Coll. No. 32 (in press).

Hauck, B. and Magnenat, P.: 1975, in A. G. D. Philip (ed.), Multicolor Photometry and the Theoretical $H R$ Diagram (in press).

Hauck, B. and van 't Veer, Cl.: 1970, Astron. Astrophys. 7, 219.

Oke, J. B. and Conti, P. S.: 1966, Astrophys. J. 143, 134.

Osawa, K.: 1965, Annals Tokyo Obs. 2nd ser. IX, No. 3.

Sandage, A. R. and Eggen, O. J.: 1969, Monthly Notices Roy. Astron. Soc. 119, 278.

Schild, R., Peterson, D. M., and Oke, J. B.: 1971, Astrophys. J. 166, 95.

\section{DISCUSSION}

Osborn: When you speak of 'subdwarfs' what do you consider as a subdwarf'? Is it a star with [lec/H] $<-0.5$, less than -1.0 , or what limit?

Hauck: The more deficient stars! With $[\mathrm{Fe} / \mathrm{H}]<-1.0$. 